\title{
Digital health and the future of diabetes management
}

\begin{abstract}
The growing expanse and interest in using mobile and wearable devices has made significant inroads into the practice of medicine and healthcare. Utilizing digital health tools in the management of diabetes is quickly becoming a large interest area by both medical researchers and companies at large. Within the last few years, there have been significant developments in tools and technology developed that could have long-lasting impact on the future of diabetes management, patient engagement and interaction, and clinical practice.
\end{abstract}

Keywords: diabetes, digital health, mobile health, wearables
Volume 2 Issue 4 - 2015

\author{
Timothy Dy Aungst,' Leonard Ming WaiTam,' \\ Dhiren Patel $^{2}$ \\ 'MCPHS University-Worcester, USA \\ ${ }^{2}$ MCPHS University-Boston, USA
}

Correspondence: Timothy Dy Aungst, MCPHS University Worcester, MA, USA, Tel 508373 5678, Fax 508756 87I5,

EmailTimothy.aungst@mcphs.edu

Received: July 21, 2015 | Published: August 12, 2015
Abbreviations: CGM, continuous glucose monitoring; FDA, food and drug administration; mHealth, mobile health; SMBG, selfmonitored blood glucose

\section{Introduction}

Digital Health is quickly becoming a high commodity item in the realm of medicine, due in no small part to the rapid advances in mobile technology in the past decade, increased access to the internet, and the potential profitability for many companies seeking to be involved in the healthcare environment. The exact nature of digital health is an evolving topic that includes in its expanse mobile devices (e.g. smartphones, tablet computers), wearable devices (e.g. fitness trackers), telemedicine utilization, and overall integration with the internet and cloud computing.

Included in the sphere of digital health is the subset topic of mobile health (mHealth) which is more focused on the use of mobile smart devices and wearables as tools in healthcare. ${ }^{2}$ Nonetheless, this field of medicine has not only captivated the interests of medical professionals and patients at large, but also enamored the corporate environment as an area to tap potential consumer base to gear new product development. . $^{3-5}$

Key interests lay upon the concomitant factors of chronic disease management and high cost of medical care, and the intercession use of data collection to monitor, manage, and make therapeutic decisions by providers for patient care..$^{6-8}$

Diabetes is one chronic disease rife with potential to use technology to innovate and disrupt current medical practices. Currently, medical practitioners are in the midst of evaluating the relevance and impact of digital health interventions on the care of patients with diabetes. ${ }^{9-16}$ Such research includes new methods of not only collecting data in real-time for therapeutic decision making, but also monitoring of concomitant disease states (e.g. hypertension), and helping patients with their medical therapy.

While this research is in a nascent space, the sphere amount of interest and early results have demonstrated potentially large ramifications to current and future diabetes management through the help of mHealth. Taking this into consideration, several developments in the mobile market are worth highlighting for those vested in diabetes care and management, which may shortly impact multiple professionals in the near future.

\section{Growth of mobile technology in diabetes}

One key area of technology being used in diabetes management is a number of mobile medical and health related apps for diabetes. Currently, there are more than 10,000 health and wellness and medical apps available across the Apple and Google app stores, with a significant number geared towards diabetes care. ${ }^{17}$

These apps range from those that deal with physical activities, diet management and those with explicit diabetes functions that allow users to track blood glucose levels and medication use. These apps not only allow a patient to track such valuable data, but also share it with their providers. Beyond this, includes a number of wearable's and mobile devices that serve as adjunctive management tools for patients with diabetes.

One of the most advanced apps to come to the market includes Roche's Accu-Check Connect diabetes management app, which has recently been approved by the Food and Drug Administration. ${ }^{18}$ The app allows patients not only to input their self-monitored glucose levels (SMBGs), but also images of their food and carb estimates of their daily intake. However, the biggest surprise in the Accu-Check Connect app is that it interprets a patient's carbohydrate intake and their SMBGs to inform the patient of how much bolus insulin they need to treat themselves using a built in algorithm. This data can in turn be overviewed and observed by a provider connected to the system to make any therapeutic decisions they feel are necessary and keep tabs on their patients.

While these apps and devices are newer to the market and still finding their role into patient care, research is ongoing to determine their benefit and widespread impact. However, this shortcoming has not stopped other agencies that are not traditionally thought to be involved in the healthcare environment from taking an interest, such as Apple and Google, who are beginning to create apps and systems to expand upon this research process. Apple has recently developed ResearchKit ${ }^{19}$ an app suite for developers looking to use their smartphones to collect data from patients for medical research. ${ }^{19}$ 
Massachusetts General Hospital is one such partner who is looking to use their ResearchKit ${ }^{19}$ app called GlucoSuccess ${ }^{20}$ for diabetes research. ${ }^{20}$

The premise behind GlucoSuccess ${ }^{20}$ is that the app will collect data from users with type 2 Diabetes, including demographic data and pertinent health data, along with their daily activities and SMBGs that the patient either inputs directly into the app or is collected from associated devices synced with the iPhone. This data will then be used by researchers at Massachusetts General Hospital in their research on diabetes and use of mobile services by patients in their care.

\section{Mobile health devices and wearables for diabetes}

Many patients and practitioners are familiar with the typical blood glucose monitors that patients utilize to perform their selfmonitored blood glucose (SMBG) measurements in the ambulatory setting. While this tried-and-true device synonymous with diabetes management has proven to be of benefit to patients with diabetes, there has been a movement to 'upgrade' these devices with mHealth innovations. The fact that recent technological developments offer the ability to not only collect, collate, and even interpret data in real-time and then be shared with providers changes the dynamic of outpatient monitoring with onsite clinical visits to one where patients can be monitored and communicated based on real-time data acquisition.

One example has included the creation of blood glucose monitors that can be directly attached or synced to a smartphone. ${ }^{21}$ These devices, allows users to test their sugars in the traditional method with blood collection and evaluation with a test strip via the blood glucose monitor, but then the data collected is maintained directly on a smartphone via the linked app. Data collected can then more accurately be timed, synced, and related back to other events occurring through the day. This then also allows the data to be uploaded or shared much easier with the user's caregiver in a data set easier for interpretation.

Similar to the previous mentioned apps incorporating gamification elements to teach your patients with diabetes to manage their health, there have been several innovative 'devices' created to help children overcome fears and learn about their diseases. One example, is a stuffed-bear that is integrated with mobile devices, that serves as a play item for children to learn about diabetes (and now allergies) by both playing with and taking care of the stuffed bear that has diabetes. ${ }^{22}$

Going beyond an actual stuffed animal for the patient to care for, there have been the creation of apps that simulate a virtual pet with diabetes for the patient to take care of on their own..$^{23}$ These mechanisms lend a patient, especially one young and new to the longterm requirement of healthcare interventions, to learn about their disease and management, and hopefully overcome their fears about treatment and become empowered about their own management.

Lastly, the use of wearables and connection with other mobile devices is leading to a new paradigm in terms of data collection in patients with diabetes. Continuous Glucose Monitoring (CGM) allows a patient to utilize sensors to track in real-time their glucose levels and fluctuations throughout the day. This is often accomplished through the application of a biosensor on the patient that tracks blood glucose concentrations, and the data is this transferred wirelessly to an external device or smartphone app. The Food and Drug Administration has already cleared several devices that perform such functions. One example includes Dexcom, whose G4 Platinum System utilizes a biosensor that is attached directly to the body that then transmits data to a wearable device on the patient which collects and records blood glucose every few minutes. ${ }^{24}$

Interestingly, Google has also demonstrated an interest in diabetes, and are actively pursuing the creation of a device that can also achieve CGM. However, unlike Dexcom's approach with a wearable biosensor on the abdomen and body, Google is looking for an unconventional route, the eye. ${ }^{25}$ Google is currently in the process of creating a 'smart-contact lens' that can detect the fluctuations in a patient's blood glucose levels via the blood vessels on the eye, and has partnered with Novartis. While this project is far from completion, it demonstrates the inventiveness of the technology industry and possible different ways that healthcare has been tackling diabetes management.

\section{Issues with integration of mhealth into diabetes management and patient care}

While many of the currently covered topics are both innovative and intriguing for patient care and utilization by clinicians, there are several concerns related to the use of mHealth technology in diabetes management. First, the issue of patient information protection is tantamount. While patients and providers may be quick to utilize digital services to supplement or replace current tools, considerations must be discussed on the security of data shared over wireless services or stored in databases online. Second, reimbursement and cost coverage of these products may be a limiting factor for patients that may be apt to utilize such products. As these products and services are relatively new, insurance companies may not reimburse or cover such products when compared to standards-of-care, and will unlikely change until data and research can demonstrate a meaningful benefit. Lastly, education of current healthcare professionals and patients on the use of these products will take time and effort, and the topic of mobile health is one that is bound to cause consternation for some individuals as they adapt to a new era of healthcare.

\section{Conclusion}

Mobile Health is paving a new way forward in the dynamic interaction between patients, providers, and mobile devices. While the use of smartphones and wearables is steadily increasing amongst members of society for personal use, the application of such technology into the management of diabetes care is quickly approaching largescale interests. Companies are starting to innovate the way patients interact with new devices to manage their care and this in turn will be used by providers and healthcare professionals in the management of their patients.

\section{Acknowledgements}

None.

\section{Conflict of interest}

Author declares that there is no conflict of interest.

\section{References}

1. Elenko E, Underwood L, Zohar D. Defining digital medicine. Nat Biotechnol. 2015;33(5):456-461.

2. National Institutes of Health. mHealth - Mobile Health Technologies.

3. IMS Institute for Healthcare Informatics. Patient Apps for Improved Healthcare: From Novelty to Mainstream. 2013. 
4. Steinhubl SR, Muse ED, Topol EJ. Can mobile health technologies transform health care?. JAMA. 2013;310(22):2395-2396.

5. Topol EJ, Steinhubl SR, Torkamani A. Digital medical tools and sensors. JAMA. 2015;313(4):353-354.

6. Bradway M, Årsand E, Grøttland A. Mobile Health: empowering patients and driving change. Trends Endocrinol Metab. 2015;26(3):114-117.

7. Anglada-martinez H, Riu-viladoms G, Martin-conde M, et al. Does mHealth increase adherence to medication? Results of a systematic review. Int J Clin Pract. 2015;69(1):9-32.

8. Chiauzzi E, Rodarte C, Dasmahapatra P. Patient-centered activity monitoring in the self-management of chronic health conditions. $B M C$ Med. 2015;13(1):77.

9. Dobson R, Carter K, Cutfield R, et al. Diabetes Text-Message SelfManagement Support Program (SMS4BG): A Pilot Study. JMIR Mhealth Uhealth. 2015;3(1):32

10. Torbjørnsen A, Jenum AK, Småstuen MC, et al. A Low-Intensity Mobile Health Intervention With and Without Health Counseling for Persons with Type 2 Diabetes, Part 1: Baseline and Short-Term Results From a Randomized Controlled Trial in the Norwegian Part of RENEWING HEALTH. JMIR Mhealth Uhealth. 2014;2(4):52.

11. Holmen H, Torbjørnsen A, Wahl AK, et al. A Mobile Health Intervention for Self-Management and Lifestyle Change for Persons With Type 2 Diabetes, Part 2: One-Year Results From the Norwegian Randomized Controlled Trial RENEWING HEALTH. JMIR Mhealth Uhealth 2014;2(4):57.

12. Tatara N, Arsand E, Skrøvseth SO, Hartvigsen G. Long-term engagement with a mobile self-management system for people with type 2 diabetes. JMIR Mhealth Uhealth. 2013;1(1):1.
13. Toledo FG, Triola A, Ruppert $\mathrm{K}$, et al. Telemedicine consultations: an alternative model to increase access to diabetes specialist care in underserved rural communities. JMIR Res Protoc. 2012;1(2):14.

14. Greenwood DA, Young HM, Quinn CC. Telehealth Remote Monitoring Systematic Review: Structured Self-monitoring of Blood Glucose and Impact on A1C. J Diabetes Sci Technol. 2014;8(2):378-389.

15. Bader A, Gougeon R, Joseph L, et al. Nutritional education through internet-delivered menu plans among adults with type 2 diabetes mellitus: pilot study. JMIR Res Protoc. 2013;2(2):41.

16. Moreau M, Gagnon MP, Boudreau F. Development of a fully automated, web-based, tailored intervention promoting regular physical activity among insufficiently active adults with type 2diabetes: integrating the I-change model, self-determination theory, and motivational interviewing components. JMIR Res Protoc. 2015;4(1):25.

17. research2guidance. mHealth App Developer Economics 2014. 2014.

18. Accu-Chek Connect. Roche. 2015.

19. ResearchKit. Apple. 2015.

20. GlucoSuccess. Build Healthy Behaviors. 2015.

21. iBGStar. SANOFI DIABETES. 2015.

22. Jerry The Bear. Sproutel. 2015.

23. Dex: Your Virtual Pet. Apple iTunes Store. 2015.

24. Dexcom. Introducing the Dexcom $\mathrm{G}^{4}$ PLATINUM system with Share ${ }^{\mathrm{TM}}$ 2015.

25. NOVARTIS. Novartis to license Google “Smart Lens” Technology. 2015. 